\title{
Agrupamento de trabalhadores com perfis semelhantes de aprendizado apoiado em Análise de Componentes Principais
}

\author{
Clustering of workers with similar learning profiles based \\ on Principal Component Analysis
}

\author{
Bárbara Brzezinski Azevedo ${ }^{1}$ \\ Michel José Anzanello'
}

\begin{abstract}
Resumo: A manufatura de produtos personalizados em larga escala, chamada customização em massa, implica o aumento da variedade de modelos e redução no tamanho dos lotes de produção. Tarefas que dependem da habilidade humana são especialmente afetadas nesse contexto, visto que os trabalhadores precisam se adaptar às características do novo modelo. Esse processo de adaptação pode ocorrer de forma distinta dentro do grupo de trabalhadores, justificando o desenvolvimento de sistemáticas com vistas ao agrupamento de indivíduos com características de aprendizado semelhantes. Este artigo propõe um método para formação de grupos homogêneos de trabalhadores de acordo com seus perfis de aprendizado pela integração de curvas de aprendizado e clusterização. Para tanto, dados de desempenho são coletados e modelados por meio de curvas de aprendizado; os parâmetros oriundos da modelagem quantificam o processo de adaptação dos trabalhadores às tarefas, servindo de base para o agrupamento dos trabalhadores. Na primeira proposição deste artigo, os dados originais (parâmetros) são clusterizados pelo método K-Means, e a qualidade do agrupamento formado, avaliada por intermédio do Silhouette Index (SI). Em uma proposição alternativa, a Análise de Componentes Principais (ACP) é aplicada sobre os dados originais e as variáveis latentes geradas (escores) são clusterizados por meio do K-Means. Quando aplicado em um processo da indústria calçadista, a clusterização apoiada nos escores apresentou um incremento na qualidade dos agrupamentos medida por SI de $147 \%$ (de SI=0,392 para $\mathrm{SI}=0,968$ ) quando comparado à clusterização aplicada às variáveis originais. Os dois grupos de trabalhadores gerados pelo método foram corroborados com base na separação em um gráfico relacionando os componentes principais obtidos. Por fim, um estudo de simulação foi realizado para corroborar a eficácia do método proposto, o qual se mostrou robusto quando submetido a diferentes níveis de ruído, correlação e proporções de variáveis e observações. Por meio da análise de variâncias (ANOVA), verificou-se que os fatores variância do ruído e proporção de observações por variáveis interferiram significativamente na qualidade da clusterização; por sua vez, a correlação entre as variáveis não apresentou variância significativa nos agrupamentos.

Palavras-chave: Curvas de aprendizado. Clusterização. Análise de componentes principais.
\end{abstract}

\begin{abstract}
The manufacturing of customized products, also called mass customization, implies increasing choice menu and reduced size of production batches. Tasks that depend on human ability are especially affected in this context, because workers need to adapt to the characteristics of new models. This adaptation process occurs differently among workers, justifying the development of systematics aiming at clustering workers with similar learning behaviors. This paper proposes a method of grouping workers according to their learning profiles, integrating learning curves (LC) and cluster analysis. To this end, performance data are collected and modeled through learning curves; parameters derived from the modeling quantify workers' adaptation to tasks. In the first scheme proposed in this study, the original data (parameters from LC modeling) are clustered using the K-Means, and clustering quality is assessed by the Silhouette Index (SI). In the second scheme, Principal Component Analysis (PCA) is applied to the original data and the latent variables (scores) are used as clustering variables using the K-Means. Clustering using the scores yielded $S I=0.968$, while grouping based on the original variables led to $S I=0.392$. A simulation study was performed to corroborate the effectiveness of the proposed method, which proved to be robust when affected by different levels of noise, correlation and different proportions of variables to observations.
\end{abstract}

Keywords: Learning curves. Clustering. Principal component analysis.

\footnotetext{
${ }^{1}$ Universidade Federal do Rio Grande do Sul -UFRGS, Av. Osvaldo Aranha, 99, CEP 90.035-190, Porto Alegre, RS, Brasil, e-mail: babibrzezinski@gmail.com; anzanello@producao.ufrgs.br
}

Recebido em Nov. 11, 2013 - Aceito em Jul. 14, 2014

Suporte financeiro: CAPES. 


\section{Introdução}

A redução do ciclo de vida dos produtos e o aumento da competição industrial fizeram com que muitas indústrias de massa repensassem suas estratégias de produção, focando as necessidades individuais dos clientes. Assim, no final dos anos 1980, surge o conceito de Customização em Massa (CM), que é baseado no fornecimento de produtos personalizados por processos flexíveis em altos volumes a custos razoavelmente baixos (SILVEIRA; BORESTEIN; FOGLIATTO, 2001), semelhantes aos custos de produtos padronizados (MACCARTHY; BRABAZON; BRAMHAM, 2003) e com integração entre os membros da cadeia de suprimentos (FOGLIATTO; SILVEIRA; BORESTEIN, 2012).

Devido a características específicas, diversos produtos customizados apoiam-se em processos de produção manuais (FOGLIATTO; SILVEIRA; BORESTEIN, 2012), sendo confeccionados em pequenos lotes (ANZANELLO; FOGLIATTO, 2011a). Esta realidade, relacionada a exigências dos consumidores por produtos diferenciados e maiores opções de catálogo, faz com que os processos produtivos alterem-se frequentemente, expondo constantemente o trabalhador à produção de novos modelos, tecnologias e processos. Tal exposição tende a reduzir os patamares de produção e níveis de qualidade dos produtos nos ciclos iniciais, devido ao processo de adaptação (aprendizado) a que o trabalhador é submetido quando da solicitação de um novo produto (NEMBHARD; UZUMERI, 2000a). Como ferramenta de auxílio à solução de alguns problemas oriundos da CM, a modelagem por Curvas de Aprendizado (CAs) vem ganhando importância no contexto produtivo.

CAs são representações matemáticas do desempenho do trabalhador em tarefas repetitivas (FIORETTI, 2007; RIED; MIRKA, 2007; ANZANELLO; FOGLIATTO, 2011a). CAs têm sido vastamente utilizadas para estudar as alterações de desempenho de trabalhadores relacionados aos efeitos do aprendizado (PLAZA; ROHLF, 2008). Assim, a modelagem de CAs pode auxiliar na gestão da produção para a estimação dos tempos de produção, prazos de entrega, desenvolvimento de estratégias de produção mais precisas (ANZANELLO; FOGLIATTO, 2011a) e classificação de grupos de trabalhadores de acordo com seus perfis de adequação a tarefas de complexidades distintas (UZUMERI; NEMBHARD, 1998; NEMBHARD; UZUMERI, 2000a; STROIEKE; FOGLIATTO; ANZANELLO, 2013a, b).

$\mathrm{O}$ agrupamento de trabalhadores em equipes tipicamente apoia-se nos tempos médios de execução de tarefas, visando minimizar a variância entre estes. Porém, em cenários de CM caracterizados por produção de lotes em tamanhos reduzidos, a formação das equipes pode ser mais eficiente considerando os perfis de aprendizado, os quais capturam o desempenho do trabalhador desde o início do processo analisado - e não somente quando o processo de aprendizado está concluído (ANZANELLO; FOGLIATTO, 2007a). Tal agrupamento permite a alocação de trabalhadores com padrões similares de aprendizado à mesma equipe, evitando gargalos nas linhas de produção e reduzindo níveis de ociosidade (ANZANELLO; FOGLIATTO, 2007a). É importante, no entanto, ressaltar que a homogeneidade nos perfis de aprendizado, tida como desejável neste artigo, pode não ser adequada em processos da indústria caracterizados por caráter inovador, em que a troca de conhecimento e novas informações são primordiais. Alinhada às proposições deste artigo, a homogeneidade/ similaridade no perfil de aprendizado/desempenho dos trabalhadores é relevante em contextos nos quais elevada repetitividade de procedimentos simples e manuais (característicos de linhas de montagem) são requeridos. Tal homogeneidade visa mitigar a ocorrência de gargalos produtivos e desbalanços na linha de produção.

Apesar da grande importância e aplicabilidade do tema, encontram-se na literatura poucos estudos que se valem das CAs para formação de grupos de trabalhadores com perfis semelhantes de aprendizado em aplicações customizadas. Uzumeri e Nembhard (1998) identificaram perfis distintos de trabalhadores em uma empresa de manufatura americana por análise dos parâmetros gerados pela modelagem das CAs, evidenciando as diferenças entre os trabalhadores para adequação nas tarefas. Wong, Cheung e Wu (2010) avaliaram o padrão de desempenho de empreiteiros submetidos a feedbacks regulares e identificaram perfis diferentes dentre os trabalhadores, os quais foram agrupados de acordo com os parâmetros da $\mathrm{CA}$. Com propósitos semelhantes, Stroieke, Fogliatto e Anzanello (2013a) propuseram um método para caracterizar diferentes grupos de trabalhadores utilizando curvas de aprendizado e técnicas de clusterização nos dados de desempenho dos trabalhadores. Porém os estudos apresentados não supriram todas as lacunas existentes na literatura referentes ao agrupamento de trabalhadores segundo seus perfis de aprendizado, principalmente com vistas à utilização de técnicas multivariadas como ferramentas de suporte.

Este artigo propõe um método para formação de grupos homogêneos de trabalhadores de acordo com seus perfis de aprendizado por meio da integração de curvas de aprendizado e clusterização. O método inicia com a coleta de dados de desempenho de trabalhadores, os quais são ajustados a modelos distintos de CAs. Os parâmetros gerados, os quais descrevem o processo de aprendizado dos trabalhadores, são remapeados por meio da Análise de Componentes Principais (ACP), visando aprimorar a qualidade do procedimento de clusterização. A qualidade de agrupamentos formados 
é avaliada pelo Índice Silhouette (SI), conforme proposto por Rousseeuw (1987).

Este artigo apresenta duas contribuições principais: (i) propõe um método de agrupamento de trabalhadores balizado por perfis de aprendizado que apresenta qualidade superior à clusterização tradicional; e (ii) permite agrupamentos nos quais há poucas observações coletadas (poucos trabalhadores) e um número elevado de variáveis (parâmetros da $\mathrm{ACP}$ ) a serem consideradas na clusterização.

O restante do artigo é organizado como segue. A seção 2 apresenta os fundamentos de CAs, ACP e técnicas para análise da qualidade de formação de agrupamentos. Na seção 3, é apresentado o método para formação de equipes, balizado pelo aprendizado dos trabalhadores. A seção 4 apresenta os resultados obtidos com o método e sua posterior aplicação em um estudo de caso na indústria calçadista. A seção 5 traz a conclusão do artigo e sugestões para pesquisas futuras.

\section{Referencial teórico}

Nesta seção, revisam-se os fundamentos das ferramentas utilizadas na sistemática proposta: CAs e técnicas de análise multivariada para formação de agrupamentos. Também se discorre brevemente sobre sistemáticas de aquisição de conhecimento.

\subsection{O processo de aprendizado}

Sistemáticas associadas à aquisição de conhecimento têm sido estudadas com objetivos distintos e sob várias perspectivas. Tipicamente, áreas relacionadas a sistemas produtivos têm se focado na modelagem desses processos com o intuito de monitorar custos e definir tempo de trabalho necessários para finalização de lotes produtivos (DAR-EL; RUBINOVITZ, 1991). Por sua vez, ciências relacionadas à psicologia e ergonomia têm estudado o processo de aprendizado com vistas à proposição de modelos para explicação do mecanismo de aquisição e retenção de conhecimento, identificando fatores que influenciam em tal processo (ANDERSON, 1982). Eyring, Johnson e Francis (1993) apontaram fatores responsáveis pelos diferentes níveis de aquisição de conhecimento e habilidade por parte do trabalhador frente a uma tarefa, sendo a familiaridade com a tarefa e a motivação do trabalhador em executá-la os fatores mais pronunciados.

A experiência anterior na tarefa em questão constitui um fator significativo no processo de aprendizado, visto que o fato de o trabalhador ter desempenhado a operação em algum momento anterior possibilita um desempenho diferenciado, principalmente nos primeiros ciclos de produção (NEMBHARD; OSOTHSILP, 2002). Nembhard e Uzumeri (2000a) afirmam que trabalhadores que já contam com experiência prévia na execução da tarefa tendem a atingir patamares mais elevados de desempenho final. Entretanto, Nembhard e Uzumeri (2000b) acrescentam que trabalhadores com elevados patamares de experiência tendem a assimilar procedimentos de maneira mais lenta, visto que há menos o que aprender.

Por sua vez, Pananiswami e Bishop (1991) avaliaram características da tarefa capazes de influenciar o processo de aprendizado. Dentre essas, a complexidade da tarefa mostrou-se a maior responsável pela diferença no desempenho de trabalhadores submetidos a novos procedimentos. Nembhard e Osothsilp (2002) corroboram tais afirmações, atestando que um aumento da complexidade da tarefa resulta em maior variabilidade dos parâmetros das CAs (indicando maiores disparidades no desempenho dos trabalhadores ao executarem tarefas complexas).

Adler e Clark (1991) afirmam que o ambiente de manufatura e os componentes que o caracterizam influenciam significativamente no processo de aprendizado. Tais componentes incluem investimentos em novas tecnologias, programas de treinamento e condições adequadas de trabalho. Para tanto, os mesmos autores propõem um modelo aditivo de CA, o qual se baseia no número de unidades produzidas, tempo de treinamento dos trabalhadores e tempo investido pela gerência na busca de melhorias no processo. Complementarmente, Vits e Gelders (2002) afirmam que a aquisição de conhecimento apoia-se em duas correntes: conhecimento induzido e conhecimento autônomo. $\mathrm{O}$ primeiro é promovido por meios que incentivem sua aquisição (programas de treinamento, inovações tecnológicas e investimentos que aumentem a produção); o segundo advém da repetição da tarefa pelo trabalhador, podendo apresentar grandes variações dentro de uma população em decorrência do desempenho particular de cada membro (SEREL et al., 2003).

Alinhados com as proposições anteriores, Smunt e Watts (2003) sugerem que os aprimoramentos verificados durante a execução de uma tarefa provêm do esforço repetitivo do trabalhador e das melhorias acerca dos meios produtivos. Terwiesch e Bohn (2001) consideram o aprendizado induzido mais eficiente do que o obtido de forma autônoma, visto que o primeiro leva em consideração a adequação do trabalhador à tarefa por outros meios que não só a acumulação de unidades produzidas. Por fim, Li e Rajagopalan (1998) também contestam o aprendizado oriundo exclusivamente da repetição manual de uma tarefa, argumentando que nã o elucida a origem do aprendizado e não fornece à gerência meios para administrar esse processo.

\subsection{Curvas de Aprendizado (CAs)}

CAs são modelos de regressão não lineares que associam o desempenho de trabalhadores (normalmente medido em número de unidades produzidas em 
determinado intervalo de operação) a características das tarefas (ANZANELLO; FOGLIATTO, 2007b). Sua utilização para a formação de agrupamentos foi proposta por Uzumeri e Nembhard (1998), Wong, Cheung e Wu (2010) e Stroieke, Fogliatto e Anzanello (2013b). Os principais modelos de CAs são classificados em: (i) potenciais, (ii) exponenciais e (iii) hiperbólicos e suas fundamentações teóricas são detalhadas na sequência. A Tabela 1 apresenta um resumo dos modelos, a descrição de seus parâmetros e suas aplicações. Detalhes dos modelos são apresentados a seguir.

O primeiro modelo potencial foi desenvolvido por Wright (1936), após observar que os custos de montagem de aviões reduziam-se em $20 \%$ a cada duplicação da quantidade de aeronaves produzidas, conforme a Equação 1. Devido à simplicidade matemática e capacidade de ajuste a dados empíricos (JABER; BONNEY; GUIFFRIDA, 2010), o modelo de Wright encontrou vasta aplicação prática: Jaber, Bonney e Guiffrida (2010) o utilizaram para analisar uma cadeia de suprimentos na qual a produção é submetida a processos de melhoria contínua, concluindo que a inclusão dos efeitos da aprendizagem no processo produtivo traz benefícios para toda a cadeia, reduzindo significativamente os custos associados ao abastecimento. Zorgios, Vlismas e Venieris (2009), utilizando o mesmo modelo de CA, verificaram que flutuações nas taxas de aprendizado provêm da variabilidade de capital humano e que os custos envolvidos em um ambiente produtivo podem ser racionalizados e monitorados por meio da análise da CA. Outras aplicações do modelo de Wright podem ser encontradas em Jaber e Glock (2013) e Yeh e Rubin (2012).

Devido a limitações em situações práticas, o modelo de Wright sofreu alterações ao do longo tempo. O modelo Stanford-b acrescenta um parâmetro ao modelo da Equação 1 para representar as unidades equivalentes à experiência do trabalhador no início do ciclo de produção; ver Equação 2 (YEH; RUBIN, 2012). Para representar processos que apresentam tarefas automatizadas, desenvolveu-se o modelo de DeJong, no qual a velocidade das operações é influenciada pelo maquinário que limita a capacidade do trabalhador em reduzir o tempo por unidade produzida, conforme a Equação 3 (NEMBHARD; UZUMERI, 2000b). Contemplando os parâmetros envolvidos nos modelos de Stanford-b e de DeJong, foi proposta a Curva $S$, a qual é baseada na suposição de que o início do processo de aprendizagem é mais gradual do que representado pelo modelo de Wright, ver Equação 4 (NEMBHARD; UZUMERI, 2000b).

Com vistas a contornar o comportamento assintótico do modelo de Wright quando repetições tendem ao infinito, o modelo de Plateau insere uma constante aditiva $C$ para descrever o estado estacionário de desempenho do trabalhador, fazendo com que o tempo/unidade tenda àquele valor quando o volume de produção for elevado, conforme a Equação 5. O estado estacionário é atingido quando o processo de aprendizado é encerrado ou quando há limitações de maquinário restringindo a melhoria de desempenho (ANZANELLO; FOGLIATTO, 2011a).

Com o objetivo de aprimorar as previsões em corridas de produção de longa duração, surgiram os modelos exponenciais. O primeiro deles, na Equação 6, foi proposto por Knecht (1974), objetivando aprimorar a modelagem de processos que possuem um elevado número de repetições. O modelo propõe a utilização combinada de funções exponenciais e potenciais, conforme apresentado na Tabela 1.

Mazur e Hastie (1978) propuseram o modelo Exponencial de 3 Parâmetros (Equação 7) para expressar a aprendizagem em três frentes: $(i)$ patamar máximo de desempenho a ser atingido pelo trabalhador quando a aquisição de conhecimento for completa; (ii) experiência prévia do trabalhador na execução da tarefa observada; e (iii) taxa de aprendizado, a qual quantifica a velocidade de aquisição de destreza (as unidades dos parâmetros são detalhadas na Tabela 1). Estudos realizados por Mazur e Hastie (1978) demonstram que o modelo apresenta ajustes deficientes quando o trabalhador é submetido a tarefas complexas e que exigem grande quantidade de novos conhecimentos. Por outro lado, o modelo adequa-se bem a situações nas quais o trabalhador possui experiência prévia (ANZANELLO; FOGLIATTO, 2007a).

Towill (1990) propôs o modelo de Tempo Constante estruturado de forma análoga ao Exponencial de 3 Parâmetros, conforme a Equação 8. O autor recomenda a utilização deste modelo quando a coleta de dados de desempenho inicia depois de uma curta adaptação do trabalhador à tarefa. Dardan, Busch e Sward (2006) utilizaram este modelo para avaliar o tempo de vida de um investimento em tecnologia, considerando os impactos do aprendizado.

Mazur e Hastie (1978) propuseram um modelo de CA que relaciona o número de unidades produzidas em conformidade com o número total de unidades produzidas. Sua descrição matemática consistiu em uma curva hiperbólica de 2 parâmetros, resultando no modelo Hiperbólico de 2 Parâmetros, conforme a Equação 9. Buscando considerar a experiência prévia do trabalhador na execução da tarefa, Mazur e Hastie (1978) propuseram a inclusão de um parâmetro $p$ na equação do modelo Hiperbólico de 2 Parâmetros, dando origem ao modelo Hiperbólico de 3 Parâmetros, conforme Equação 10. Tal modelo tem sido amplamente aplicado em cenários produtivos: Wong, Cheung e Hardcastle (2007) utilizaram o modelo para prever o desempenho de contratantes durante um projeto de construção civil, enquanto que 


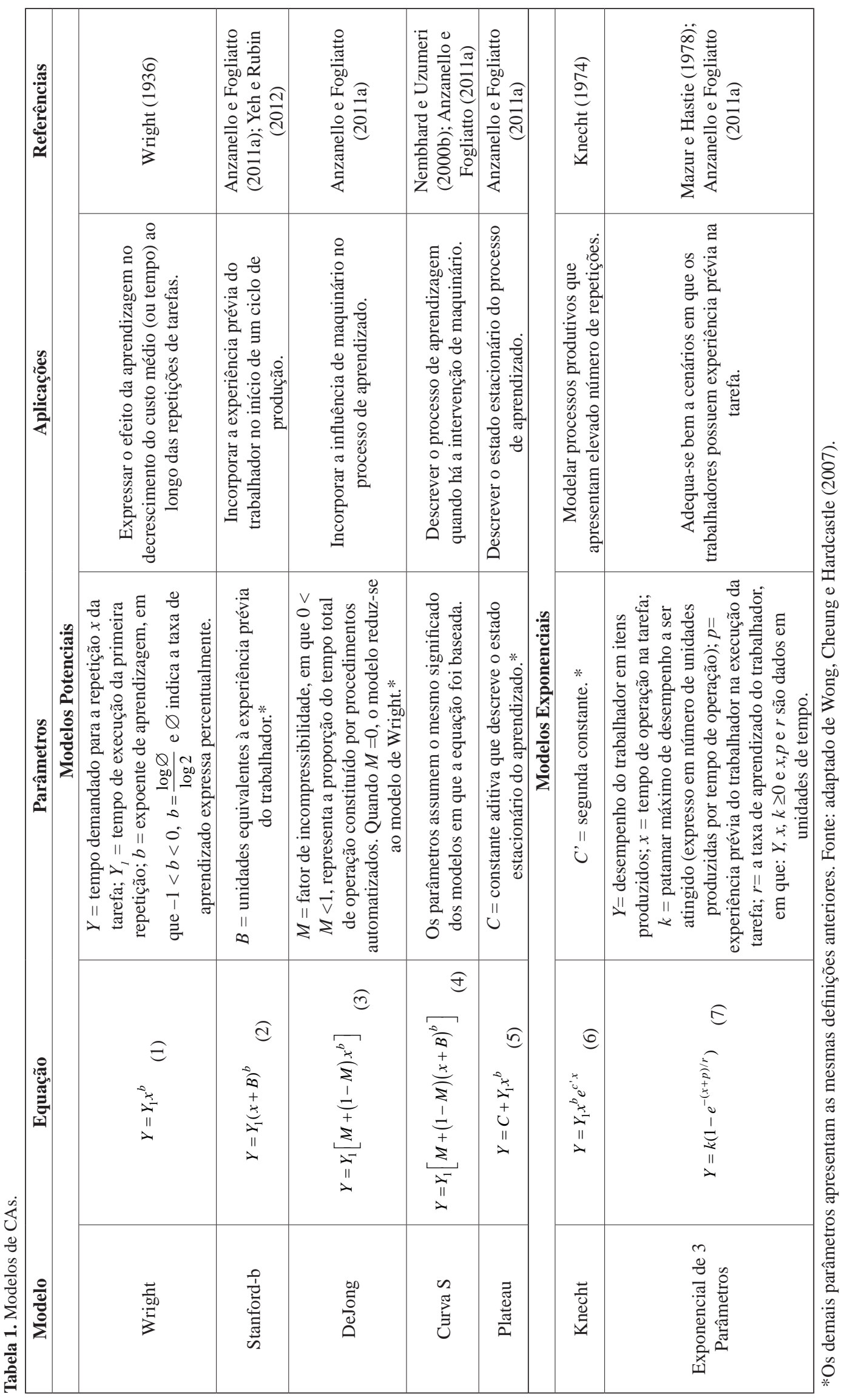




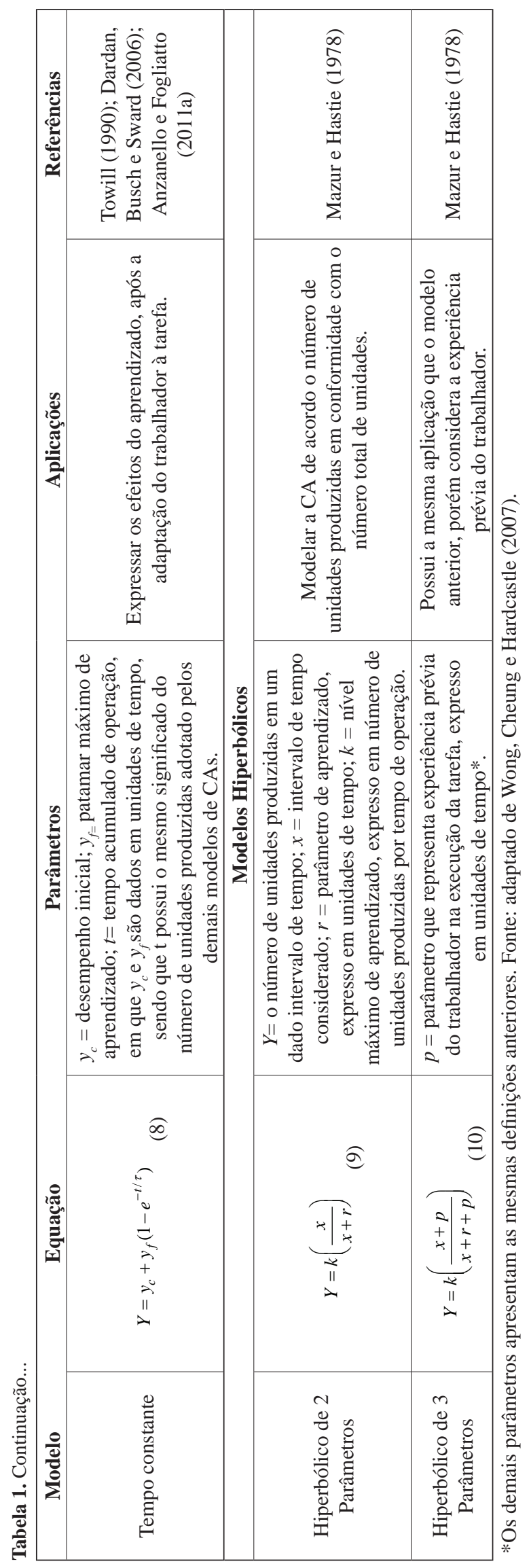


Guimarães, Anzanello e Renner (2012) obtiveram uma redução significativa no número de acidentes e no índice de absenteísmo em uma indústria de calçados, utilizando uma metodologia de rotação de trabalhadores a partir da aplicação do modelo Hiperbólico de 3 Parâmetros.

\subsection{Técnicas de análise multivariada}

Esta seção revisa as duas técnicas de análise multivariada utilizadas neste artigo: $(i)$ análise de componentes principais; e (ii) análise de agrupamentos (clustering).

\subsubsection{Análise de Componentes Principais (ACP)}

A ACP é um método utilizado para reduzir a dimensionalidade de uma matriz de dados, preservando a maior parte da variabilidade existente no conjunto de dados (RENCHER, 1995). Sua operacionalização ocorre por meio do cálculo dos autovalores e dos autovetores da matriz de covariância dos dados originais (RENCHER, 1995; JOLLIFFE, 2002). Os componentes principais (CPs) resultantes, $z$, são combinações lineares das variáveis originais, $z=a_{1} y_{1}+a_{2} y_{21}+\ldots+a_{n} y_{n}$, e são mutuamente ortogonais (RENCHER, 1995). Devido à ortogonalidade, é possível examinar as relações entre o conjunto de dados e as variáveis pelos gráficos dos escores $\left(z a_{n}\right) \mathrm{e}$ dos pesos (w), cujos eixos são CPs nos quais os dados são projetados. Os escores fornecem a composição dos CPs em relação às amostras de dados, enquanto que os pesos fornecem a mesma composição em relação às variáveis, pois os pesos decorrem da maximização da variância entre as variáveis latentes $z$ (JOLLIFFE, 2002). O número de CPs a ser retido pode ser definido com base no percentual de variância explicado por cada componente. Assim, somam-se os autovalores - arranjados do maior ao menor - até atingir o valor esperado de variância (RENCHER, 1995); pode-se também utilizar o Scree Graph nesta análise (RENCHER, 1995).

Dentre suas diversas aplicações, Mao, Hu e Song (2009) utilizaram a ACP para estruturar um questionário em componentes, buscando investigar os fatores em cada componente que influenciam na indústria automotiva chinesa, enquanto que Engblom et al. (2012) fizeram uso da ACP para agrupar custos logísticos em componentes e verificar a influência destes nos custos totais de companhias finlandesas. Lu et al. (2011) utilizaram CPs e análise de conglomerados para agrupar estações de monitoramento da qualidade do ar com base nos níveis de poluição em Hong Kong, verificando que a utilização combinada dos dois métodos possui elevada aplicação na gestão de sistemas com muitas variáveis. Gong et al. (2012) também utilizaram os dois métodos a fim de avaliar as relações existentes entre a concentração de elementos químicos presentes na substância própolis. Os métodos permitiram a formação de agrupamentos homogêneos e maior conhecimento sobre as variações das características observadas nas substâncias entre as áreas estudadas.

\subsubsection{Análise de agrupamentos}

A análise de agrupamentos ou análise de conglomerados (cluster analysis) é um método estatístico que permite agrupar observações em grupos homogêneos em função do grau de similaridade entre as observações (HAIR JUNIOR et al., 1998; FÁVERO et al., 2009). Os agrupamentos resultantes devem possuir alta homogeneidade interna e alta heterogeneidade externa; quando representados graficamente, as observações dentro dos agrupamentos estarão próximas, enquanto que as observações de diferentes grupos estarão distantes (HAIR JUNIOR et al., 1998). Na análise de agrupamentos, a variável estatística de agrupamento (conjunto de variáveis que representam as características utilizadas para comparar observações) é especificada pelo pesquisador, não sendo estimada empiricamente (HAIR JUNIOR et al., 1998; FÁVERO et al., 2009).

A análise de clusterização demanda que se determine o procedimento para agrupamento (hierárquico ou não hierárquico). Os procedimentos hierárquicos envolvem a construção de uma estrutura do tipo árvore, denominada dendograma (HAIR JUNIOR et al., 1998); neste procedimento não é necessário determinar previamente o número de clusters. Os procedimentos não hierárquicos, por sua vez, demandam a definição do número inicial de clusters para inserção das observações em grupos (FÁVERO et al., 2009). O método $K$-Means é um dos procedimentos não hierárquicos mais conhecidos (NALDI et al., 2011) e utiliza a distância euclidiana como critério para formação de grupos (FÁVERO et al., 2009). É considerado um método simples e pode ser aplicado a um grande conjunto de dados (NALDI et al., 2011). O K-Means é operacionalizado com base nos seguintes procedimentos: (i) partição inicial dos indivíduos em $K$ clusters definidos pelo pesquisador; (ii) cálculo dos centroides para cada cluster e cálculo da distância euclidiana dos centroides para cada observação da base de dados; (iii) agrupamento das observações aos clusters cujos centroides sejam mais próximos; e (iv) voltar ao passo (ii) até que não ocorra variação significativa na distância mínima de cada observação a cada um dos centroides dos $K$ clusters (NALDI et al., 2011).

Para medir a qualidade dos agrupamentos formados, Rousseeuw (1987) apresenta o índice Silhouette (SI), que mede o grau de similaridade de uma observação 
em relação às demais em seu próprio grupo, comparada às observações alocadas ao grupo mais próximo a ela. O SI é calculado pela Equação 11, em que $a$ (i) é a média da distância da $i$-ésima observação a todas as demais pertencentes ao grupo no qual a observação foi alocada e $b(i)$ é a média da distância entre a $i$-ésima observação a todas as observações do grupo vizinho mais próximo.

$$
S I=\frac{b(i)-a(i)}{\max \{b(i), a(i)\}}
$$

Os valores de SI estão no intervalo de -1 a 1 , sendo que valores próximos a -1 indicam que a observação foi erroneamente inserida no grupo de destino; valores próximos a zero denotam que a observação poderia estar tanto em seu grupo de destino quanto em algum outro grupo; e valores próximos a 1 indicam que a observação está corretamente alocada em seu grupo (ROUSSEEUW, 1987). Dentre as suas aplicações, o SI foi utilizado por Brida, Disegna e Osti (2012) para selecionar o melhor método de agrupamento de turistas no mercado italiano, enquanto que Jung e Koh (2009) o utilizaram para medir a qualidade de agrupamentos, propondo uma metodologia para a classificação e localização de danos estruturais em engenharia. Saha et al. (2011) aplicaram o SI para a avaliação do desempenho de algoritmos de clusterização criados para encontrar grupos de genes. Por fim, Stroieke, Fogliatto e Anzanello (2013a) valeram-se do SI para avaliação da qualidade dos agrupamentos de trabalhadores formados a partir dos dados de desempenho obtidos por CAs.

\section{Metodologia proposta}

O método proposto visa agrupar equipes de trabalhadores de acordo com seus perfis de aprendizado em ambientes de CM. O tempo de processamento das tarefas depende da velocidade de aprendizado dos trabalhadores e, por consequência, impacta na formação de equipes. A Figura 1 representa as etapas do método proposto, as quais são detalhadas na sequência. A sistemática proposta apoia-se em dois processos distintos de clusterização: Análise de Agrupamentos sobre Variáveis de Clusterização $p$ (AAV) e Análise de Agrupamentos sobre Componentes Principais (AACP).

\subsection{Passo 1: seleção dos trabalhadores}

Este passo inicia com a seleção de $I(i=1, \ldots I)$ trabalhadores que terão seus processos de aprendizado monitorados. É desejado que cada trabalhador esteja familiarizado com as operações. No momento em que o trabalhador é apresentado a uma nova tarefa, deve-se cronometrar o tempo demandado para execução de cada repetição. Os dados de desempenho devem ser coletados até que não sejam observadas maiores alterações no padrão de desempenho, tipicamente observado quando o número de itens produzidos em determinado intervalo de operação oscila em torno de um valor médio observado diretamente do processo.

\subsection{Passo 2: obtenção dos parâmetros para cada modelo de CA}

Os dados de desempenho dos trabalhadores são então modelados por meio das CAs apresentadas na Tabela 1. Os parâmetros das CAs podem ser estimados por rotinas disponíveis em aplicativos de análise estatística. Assim, cada trabalhador $i$ é caracterizado por um conjunto de parâmetros estimados a partir dos dados de desempenho obtidos em cada CA. Tais parâmetros são normalizados para evitar efeitos de escala no processo de agrupamento. Os parâmetros passam a ser considerados variáveis de clusterização $p$.

\subsection{Passo 3: obtenção dos componentes principais por meio dos parâmetros das CAs}

Neste passo, aplica-se a ACP sobre os parâmetros, que resultará em variáveis latentes $z$. O número de CPs a ser retido pode ser definido com base no Scree Graph (RENCHER, 1995). Depois da conclusão deste passo, haverá duas opções de variáveis de clusterização: $p$ (parâmetros originais) e $z$ (parâmetros no formato de variáveis latentes).

\subsection{Passo 4: geração do dendograma por meio da clusterização hierárquica das variáveis $p$ e $z$}

Iniciam-se duas sistemáticas paralelas de agrupamento dos trabalhadores: AAV e AACP. No processo AAV, estima-se o número recomendado de agrupamentos de acordo com o dendograma resultante e executa-se a clusterização hierárquica utilizando as variáveis $p$ por meio do método $K$-Means. A combinação dos métodos hierárquicos e não hierárquicos é feita para se obter os benefícios de ambos os métodos: o método hierárquico permite estimar um número adequado de agrupamentos de acordo com as características das observações avaliadas, sem que o pesquisador tenha de fazer esta escolha previamente, como ocorre no procedimento não hierárquico. Porém, a precisão de agrupamento é maior em métodos não hierárquicos (FÁVERO et al., 2009). Na segunda sistemática de clusterização, AACP, extraem-se variáveis latentes $z$ dos parâmetros de aprendizado para então realizar o agrupamento sobre tais variáveis, o que pode reduzir o efeito de correlação das variáveis sobre a clusterização. Na sequência, executa-se a clusterização hierárquica sobre 


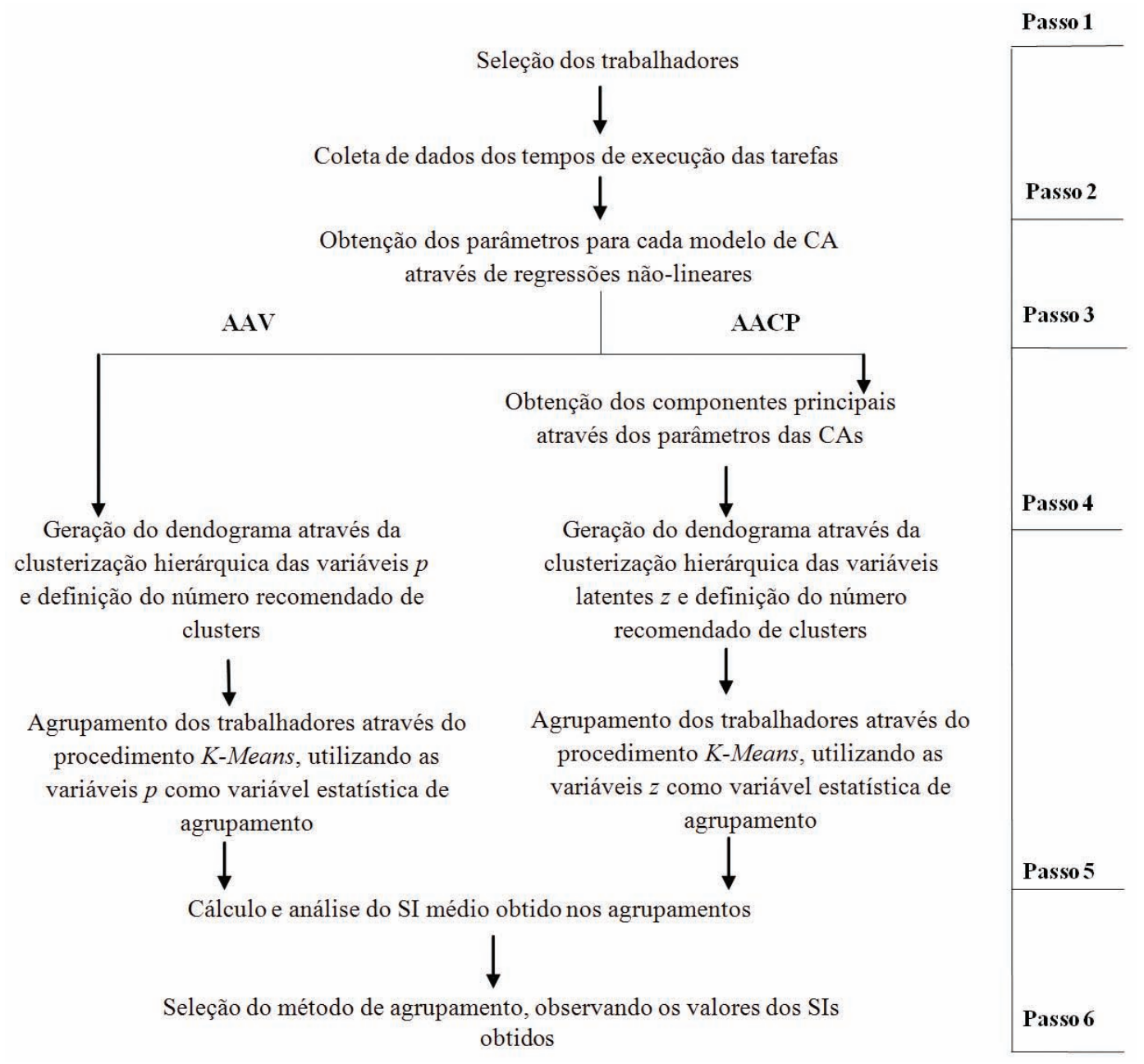

Figura 1. Passos do método proposto.

as variáveis $z$ e verifica-se o número recomendado de agrupamentos de acordo com o dendograma resultante. O número estimado de agrupamentos obtido é gerado por meio do método $K$-Means utilizando as variáveis latentes $z$.

\subsection{Passo 5: cálculo e análise do SI médio obtido nos agrupamentos}

A qualidade dos agrupamentos gerados é avaliada pela média do SI dos I trabalhadores considerados. Devem ser gerados dois Sis médios, um para a sistemática AAV e outro para AACP. Os resultados obtidos são armazenados para comparações posteriores.

\subsection{Passo 6: seleção do método de agrupamento}

Por fim, comparam-se os valores de SI obtidos no passo anterior. O objetivo é identificar a sistemática de clusterização responsável pelo maior SI, a qual é recomendada para futuros procedimentos de clusterização.

\section{Aplicação em um processo da indústria calçadista}

O método proposto foi aplicado em um processo da indústria calçadista. O setor calçadista é caracterizado por uma crescente tendência de CM evidenciada pela redução no tamanho dos lotes de produção e alternância frequente de modelos. Independente do modelo em produção, o setor de costura constitui-se no gargalo do processo produtivo, fazendo com que a produção seja dependente da habilidade manual dos trabalhadores (ANZANELLO; FOGLIATTO, 2011b).

Dados de desempenho de 21 trabalhadores foram coletados e modelados de acordo com as equações da Tabela 1. Foram gerados 29 parâmetros, apresentados no Apêndice, que representam os perfis de aprendizado dos trabalhadores. Tais parâmetros foram padronizados e utilizados como variáveis de clusterização $p$, de 


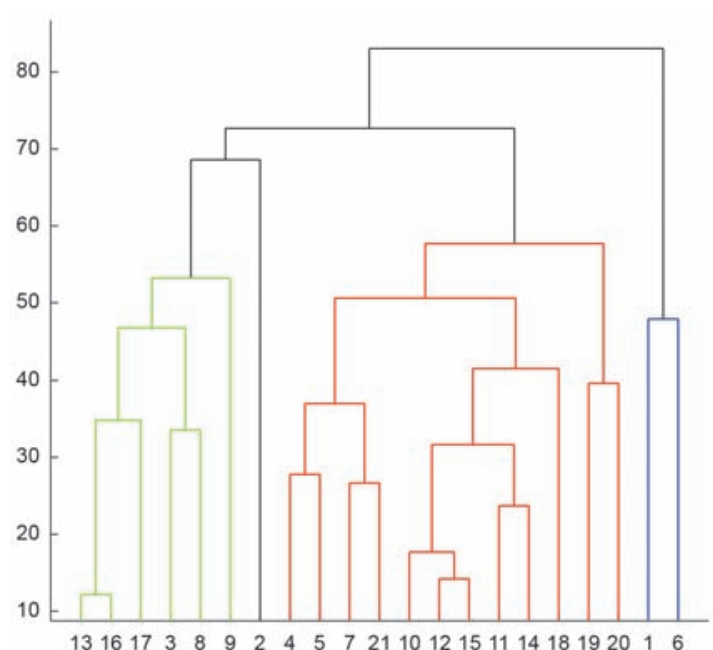

Figura 2. Dendograma AAV.

Tabela 2. Índices Silhouette Médios para os métodos AAV e AACP (de acordo com o número de componentes retidos para o AACP).

\begin{tabular}{cccc}
\hline $\begin{array}{c}\text { Número de } \\
\text { Componentes } \\
\text { Principais Retidos }\end{array}$ & $\begin{array}{c}\text { Variância } \\
\text { Acumulada } \\
\text { Explicada }\end{array}$ & AACP & AAV \\
\hline 1 & $75,00 \%$ & 0,968 & 0,392 \\
2 & $89,98 \%$ & 0,921 & \\
3 & $95,74 \%$ & 0,906 & \\
4 & $97,80 \%$ & 0,902 & \\
5 & $98,82 \%$ & 0,899 & \\
6 & $99,36 \%$ & 0,898 & \\
\hline
\end{tabular}

acordo com os passos 1 e 2 descritos acima. $\mathrm{Na}$ sequência, aplicou-se a ACP sobre os parâmetros originais, gerando as variáveis latentes $z$. Para executar a sistemática AAV, aplicou-se uma rotina de clusterização hierárquica sobre as variáveis $p$; o dendograma gerado sugeriu a formação de 4 clusters, conforme a Figura 2. Na sequência, as mesmas variáveis foram clusterizadas pelo método $K$-Means, utilizando $K=4$. O valor de SI médio resultante na AAV foi de 0,3917, conforme a Tabela 2.

A seguir, as variáveis latentes $z$ foram utilizadas para dar início à AACP. A clusterização hierárquica foi realizada para estimar o número ideal de clusters. O dendograma resultante sugere a formação de 2 clusters, conforme a Figura 3. A partir dos CPs gerados, utilizaram-se as variáveis latentes $z$ para proceder ao método $K$-Means, utilizando $K=2$. O valor de SI médio resultante na AACP foi de 0,968 , retendo-se um componente principal, explicando $75 \%$ da variância na análise, conforme Tabela 2.

A Tabela 2 apresenta a média dos valores de SIs obtidos pelos 21 trabalhadores agrupados nos dois métodos analisados. Os valores médios de SIs da

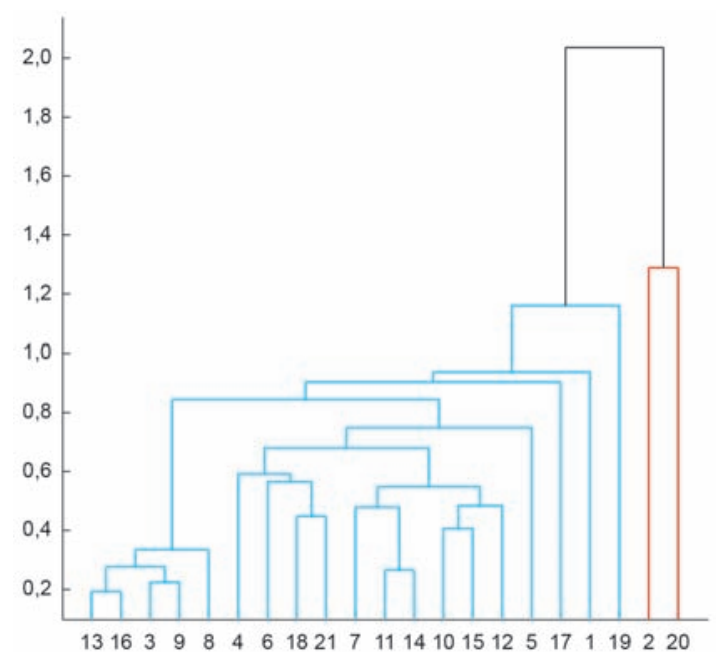

Figura 3. Dendograma AACP.

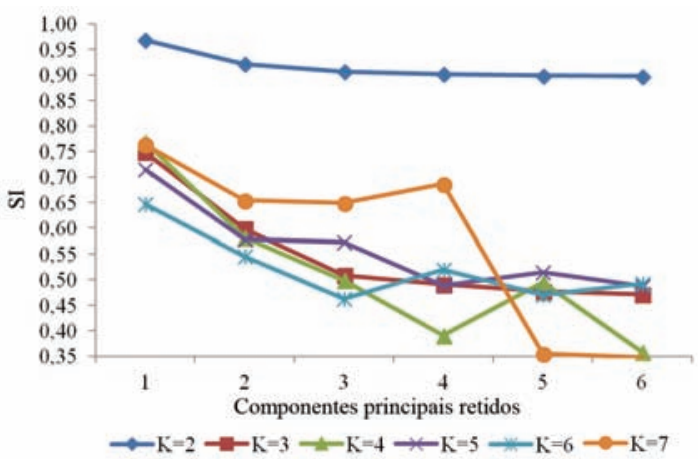

Figura 4. Variação dos Índices Silhoutte Médios para distintos $K$ s e CPs.

AACP foram obtidos fixando-se $K=2$ e alterando-se o número de componentes principais retidos na análise 2. Com base em tais resultados, o método de agrupamento recomendado é a AACP com retenção de $1 \mathrm{CP}$, o qual gera o maior SI médio.

A Figura 4 apresenta a variação nos valores médios de SIs para diferentes valores de $K$ e componentes principais retidos. Percebe-se que, para os dados em análise, a retenção de $1 \mathrm{CP}$ e a utilização de 2 clusters na análise geram o maior valor de SI. Tal resultado corrobora a sugestão de 2 clusters indicada pelo dendograma da Figura 3. Percebe-se ainda uma tendência de redução na qualidade de clusterização com a inclusão de maior número de componentes principais, o que pode ser justificado pela inserção de variáveis latentes de menor importância no procedimento de clusterização (o que acaba por comprometer a estrutura dos clusters formados).

A AACP permitiu a formação de agrupamentos homogêneos, conforme valores individuais de SI na Tabela 3. Os trabalhadores 1, 3, 4, 5, 6, 7, 8, 9, 10, $11,12,13,14,15,16,17,18,19$ e 21 foram inseridos no cluster 1 , ao passo que os trabalhadores 2 e 20 
Tabela 3. Cluster de destino dos trabalhadores e SI para cada trabalhador por meio da AACP.

\begin{tabular}{ccc}
\hline Trabalhador & Cluster & SI \\
\hline $\mathbf{1}$ & 1 & 0,983 \\
$\mathbf{2}$ & 2 & 0,986 \\
$\mathbf{3}$ & 1 & 0,964 \\
$\mathbf{4}$ & 1 & 0,985 \\
$\mathbf{5}$ & 1 & 0,943 \\
$\mathbf{6}$ & 1 & 0,984 \\
$\mathbf{7}$ & 1 & 0,968 \\
$\mathbf{8}$ & 1 & 0,976 \\
$\mathbf{9}$ & 1 & 0,961 \\
$\mathbf{1 0}$ & 1 & 0,983 \\
$\mathbf{1 1}$ & 1 & 0,976 \\
$\mathbf{1 2}$ & 1 & 0,984 \\
$\mathbf{1 3}$ & 1 & 0,969 \\
$\mathbf{1 4}$ & 1 & 0,976 \\
$\mathbf{1 5}$ & 1 & 0,959 \\
$\mathbf{1 6}$ & 1 & 0,975 \\
$\mathbf{1 7}$ & 1 & 0,944 \\
$\mathbf{1 8}$ & 1 & 0,985 \\
$\mathbf{1 9}$ & 1 & 0,862 \\
$\mathbf{2 0}$ & 2 & 0,983 \\
$\mathbf{2 1}$ & 1 & 0,983 \\
& SI médio & $\mathbf{0 , 9 6 8}$ \\
\hline
\end{tabular}

compõem o cluster 2 . Tendo em vista que todos os trabalhadores geraram SI próximo a 1, pode-se afirmar que a clusterização foi adequadamente realizada.

Os clusters apresentados na Tabela 3 são ilustrados na Figura 5, na qual se utilizam os três primeiros CPs para geração de um gráfico tridimensional. Como os CPs resultantes são combinações lineares dos parâmetros de aprendizado, os aglomerados formados em cada gráfico permitem identificar dois grupos de trabalhadores com perfis distintos de aprendizado.

As proposições deste artigo foram comparadas com o método de Uzumeri e Nembhard (1998). Naquele estudo, no entanto, a formação dos agrupamentos é feita de maneira visual e subjetiva, utilizando um gráfico tridimensional gerado a partir dos parâmetros $k, p$ e $r$ oriundos da CA hiperbólica de 3 parâmetros. Tal gráfico foi gerado para os dados deste artigo (e não apresentado por restrições de espaço), porém os clusters formados pela proposição de Uzumeri e Nembhard (1998) pareceram muito menos conclusivos do que os gerados pelo método deste artigo; justifica-se tal resultado pelo fato de os clusters desse estudo apoiarem-se em escores da ACP gerados a partir de 29 parâmetros de distintos modelos de curvas (e não somente 3, como em UZUMERI; NEMBHARD, 1998).

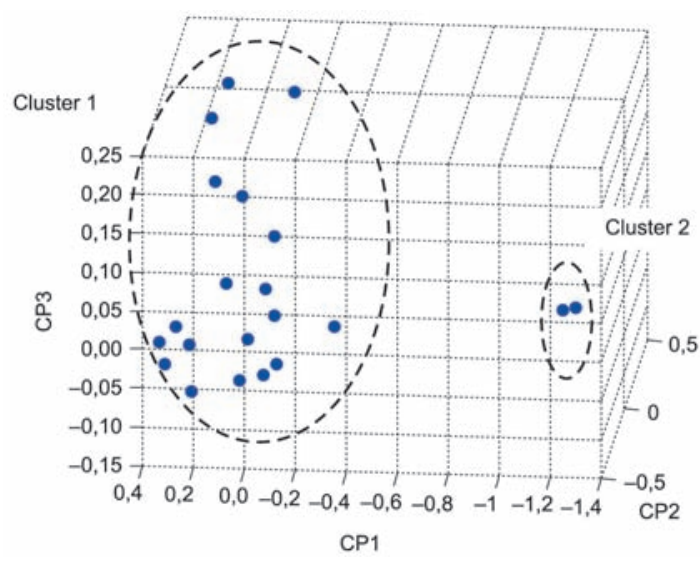

Figura 5. Agrupamentos gerados pelas variáveis latentes oriundas de $3 \mathrm{CPs}$ retidos.

Tabela 4. Fatores e níveis do experimento de simulação.

\begin{tabular}{lc}
\hline \multicolumn{1}{c}{ Fator } & Nível \\
\hline Variância do ruído & {$[0 ; 0,1],[0 ; 0,2],[0,1 ; 0,4]$} \\
Correlação dos parâmetros & $\Gamma^{3} ; \Gamma$ \\
Proporção de observações & 0,$72 ; 1 ; 7,2$ \\
por variáveis & \\
\hline
\end{tabular}

\section{Experimentos de simulação}

Devido ao satisfatório desempenho gerado pelo método AACP frente ao AAV no estudo aplicado em dados industriais, realizaram-se experimentos de simulação com o objetivo de avaliar o comportamento do método diante da inclusão de fatores como ruído, correlação nos dados e diferentes proporções entre o número de variáveis e de observações. A seguir, apresenta-se o projeto do experimento de simulação e os resultados obtidos.

\subsection{Projeto da simulação}

No estudo de caso da seção 4, AACP apresentou maior desempenho com a retenção de $1 \mathrm{CP}$ e a utilização de $K=2$ clusters. Sendo assim, estes fatores foram mantidos fixos na simulação para avaliação do método. Todas as simulações são baseadas no conjunto de dados utilizado no estudo de caso. Os dados originais possuem 29 variáveis (parâmetros de aprendizado) e 21 observações, que correspondem ao número de trabalhadores analisados. Os experimentos foram realizados em Matlab 7.8.

Os dados foram gerados por uma distribuição multinormal, combinando os níveis de três fatores analisados no experimento, apresentados na Tabela 4. O nível nominal de variância do ruído gera vetores de médias obtidos por meio de uma distribuição uniforme no intervalo $[0 ; 0,4]$. O nível baixo corresponde a $[0 ; 0,1]$, enquanto o nível alto a $[0,1 ; 0,4]$. Tais níveis foram definidos de forma empírica. Para o fator de 
correlação, foi gerada a matriz de correlação $(\Gamma)$ a partir dos dados originais. $\Gamma^{3}$ corresponde ao nível baixo e $\Gamma$ ao nível alto. A potência ímpar foi utilizada para preservar o sinal das correlações entre as variáveis. Há três níveis de proporção entre observações e variáveis: (i) razão de 0,72 (21 observações /29 variáveis), denotando o nível baixo; (ii) razão de 1,0 (29 observações/29 variáveis), denotando o nível médio; e (iii) razão de 7,2 (210/29), correspondendo ao nível alto. Foram gerados $3 \times 2 \times 3=18$ cenários

Tabela 5. Resultados da simulação para a escolha do melhor método de agrupamento.

\begin{tabular}{|c|c|c|c|c|c|c|}
\hline \multirow{2}{*}{$\begin{array}{c}\text { Proporção de } \\
\text { Observações } \\
\text { por Variáveis }\end{array}$} & \multirow[t]{2}{*}{ Correlação } & \multirow[t]{2}{*}{ Ruído } & \multicolumn{2}{|c|}{$\mathbf{A A C P}$} & \multicolumn{2}{|c|}{ AAV } \\
\hline & & & SI média & SI desvio & SI média & SI desvio \\
\hline \multirow{6}{*}{0,72} & \multirow{3}{*}{$\Gamma^{3}$} & {$[0 ; 0,1]$} & 0,7417 & 0,0684 & 0,2826 & 0,0590 \\
\hline & & {$[0 ; 0,2]$} & 0,7373 & 0,0698 & 0,2800 & 0,0569 \\
\hline & & {$[0,1 ; 0,4]$} & 0,7390 & 0,0694 & 0,2809 & 0,0553 \\
\hline & \multirow{3}{*}{$\Gamma$} & {$[0 ; 0,1]$} & 0,7391 & 0,0713 & 0,3528 & 0,0671 \\
\hline & & {$[0 ; 0,2]$} & 0,7401 & 0,0702 & 0,3539 & 0,0714 \\
\hline & & {$[0,1 ; 0,4]$} & 0,7396 & 0,0712 & 0,3543 & 0,0720 \\
\hline \multirow{6}{*}{1} & \multirow{3}{*}{$\Gamma^{3}$} & {$[0 ; 0,1]$} & 0,7304 & 0,0571 & 0,3030 & 0,0522 \\
\hline & & {$[0 ; 0,2]$} & 0,7243 & 0,0588 & 0,2815 & 0,0505 \\
\hline & & {$[0,1 ; 0,4]$} & 0,7386 & 0,0583 & 0,2676 & 0,0447 \\
\hline & \multirow{3}{*}{$\Gamma$} & {$[0 ; 0,1]$} & 0,7317 & 0,0591 & 0,3601 & 0,0596 \\
\hline & & {$[0 ; 0,2]$} & 0,7316 & 0,0582 & 0,3607 & 0,0570 \\
\hline & & {$[0,1 ; 0,4]$} & 0,7264 & 0,0591 & 0,3375 & 0,0578 \\
\hline \multirow{7}{*}{7,2} & \multirow{3}{*}{$\Gamma^{3}$} & {$[0 ; 0.1]$} & 0,7213 & 0,0203 & 0,2559 & 0,0168 \\
\hline & & {$[0 ; 0.2]$} & 0,7054 & 0,0211 & 0,2460 & 0,0151 \\
\hline & & {$[0,1 ; 0.4]$} & 0,7129 & 0,0215 & 0,2757 & 0,0172 \\
\hline & \multirow{4}{*}{$\Gamma$} & {$[0 ; 0.1]$} & 0,7112 & 0,0211 & 0,3013 & 0,0200 \\
\hline & & {$[0 ; 0.2]$} & 0,7140 & 0,0204 & 0,3260 & 0,0200 \\
\hline & & {$[0,1 ; 0.4]$} & 0,7149 & 0,0207 & 0,3162 & 0,0202 \\
\hline & & Média & 0,73 & 0,05 & 0,31 & 0,05 \\
\hline
\end{tabular}

Tabela 6. Resultados ANOVA para nível de significância dos fatores.

\begin{tabular}{|c|c|c|c|c|c|c|}
\hline Fator & $\begin{array}{c}\text { Soma } \\
\text { quadrada }\end{array}$ & $\begin{array}{l}\text { Graus de } \\
\text { liberdade }\end{array}$ & $\begin{array}{c}\text { Média } \\
\text { Quadrada }\end{array}$ & $\mathbf{F}$ & $\begin{array}{l}\text { Probabilidade } \\
\text { F }\end{array}$ & Significância \\
\hline \multicolumn{7}{|c|}{ AACP } \\
\hline Ruído & 0,00005 & 2 & 0,00002 & 1,34 & 0,2978 & Significativo \\
\hline $\begin{array}{l}\text { Correlação dos } \\
\text { parâmetros }\end{array}$ & 0 & 1 & 0 & 0,02 & 0,9011 & $\begin{array}{c}\text { Não } \\
\text { Significativo }\end{array}$ \\
\hline $\begin{array}{l}\text { Proporção de } \\
\text { Observações } \\
\text { por variáveis }\end{array}$ & 0,00212 & 2 & 0,00106 & 58,28 & 0 & Significativo \\
\hline Erro & 0,00022 & 12 & 0,00002 & & & \\
\hline Total & 0,00239 & 17 & & & & \\
\hline \multicolumn{7}{|c|}{ AAV } \\
\hline Ruído & 0,00005 & 2 & 0,00002 & 0,15 & 0,8647 & $\begin{array}{c}\text { Não } \\
\text { Significativo }\end{array}$ \\
\hline $\begin{array}{l}\text { Correlação dos } \\
\text { parâmetros }\end{array}$ & 0,01931 & 1 & 0,01931 & 118,56 & 0 & Significativo \\
\hline $\begin{array}{l}\text { Proporção de } \\
\text { Observações } \\
\text { por variáveis }\end{array}$ & 0,00386 & 2 & 0,00193 & 11,85 & 0,0014 & Significativo \\
\hline Erro & 0,00195 & 12 & 0,00016 & & & \\
\hline Total & 0,02518 & 17 & & & & \\
\hline
\end{tabular}


diferentes para cada método de agrupamento (AACP e AAV) e simuladas 1.000 repetições para cada cenário.

\subsection{Resultados da simulação}

A Tabela 5 apresenta a média e desvio padrão das 1000 repetições para cada cenário avaliado. Percebe-se que a AACP apresenta desempenho superior frente à $\mathrm{AAV}$ quando da inserção de ruído, correlação e diferentes proporções de observações por variáveis, obtendo um SI médio de 0,73 e desvio padrão de 0,05 contra $\mathrm{SI}=0,31$ e desvio=0,05 apresentado pelo AAV.

A simulação comprova a robustez do AACP, que utiliza as variáveis latentes para agrupar as observações frente à utilização das variáveis originais utilizadas pelo AAV. Tal resultado decorre da redução do efeito de correlação das variáveis sobre a clusterização obtida pelo uso dos componentes como variáveis de clusterização.

Por meio da análise de variâncias (ANOVA), pode-se avaliar o nível de significância dos fatores na simulação. Admitindo-se um nível de significância de $5 \%$, verificou-se que os fatores variância do ruído e proporção de observações por variáveis interferem significativamente nos resultados do AACP, já a correlação entre as variáveis não apresentou variância significativa sobre a clusterização, conforme a Tabela 6. A não significância do fator de correlação era esperada, visto que a ACP utilizada no método AACP gera componentes ortogonais (sem correlação) para clusterização.

Para a AAV, os fatores correlação entre as variáveis e proporção de observações por variáveis foram significativos nos resultados.

\section{Conclusões}

Este artigo apresentou um método para a formação de grupos homogêneos de trabalhadores de acordo com seus perfis de aprendizado que integra curvas de aprendizado e análise de agrupamentos. As curvas são utilizadas para a geração de parâmetros que descrevem características dos perfis de aprendizado dos trabalhadores; tais parâmetros são avaliados por meio da Análise de Componentes Principais (ACP) com vistas à melhoria da precisão da clusterização. A qualidade dos agrupamentos formados foi avaliada pelo Índice Silhouette (SI).

Ao ser aplicado em um processo da indústria calçadista, o método em que os parâmetros são transformados em componentes principais apresentou um índice Silhouette 0,968, valor superior a 0,392 obtido com a clusterização tradicional. Além disso, o método proposto permitiu gerar agrupamentos nos quais há poucas observações disponíveis (21 trabalhadores observados, e um número superior de variáveis a serem consideradas na clusterização, 29 parâmetros de aprendizado), cenário este que pode comprometer a eficiência de algumas ferramentas multivariadas, visto que a estimativa das distâncias entre as observações e posterior agrupamento poderia ser afetada pela presença de grande número de variáveis ruidosas que pouco contribuem com a estruturação dos clusters. Para corroborar a robustez do método, foi realizado um experimento de simulação, o qual comprovou o desempenho superior do AACP frente ao AAV, mesmo com a inserção de fatores de ruído, correlação e diferentes proporções de variáveis por observações nos agrupamentos formados. Pela análise de variâncias (ANOVA), verificou-se que os fatores variância do ruído e proporção de observações por variáveis interferiram significativamente na qualidade da clusterização; por sua vez, a correlação entre as variáveis não apresentou variância significativa nos agrupamentos.

Em termos práticos, a aplicação do método proposto no processo calçadista em análise visa minimizar os impactos decorrentes de distintos perfis de aprendizado e desempenho dos trabalhadores na composição de linhas de produção, especificamente na linha de costura (alvo deste estudo). Espera-se que os agrupamentos propostos conduzam a menor desbalanço nos tempos de operação nas posições da linha, gerando ritmos homogêneos de produção.

Trabalhos futuros visam à construção de uma sistemática para seleção das variáveis (parâmetros de aprendizado) que serão utilizadas na clusterização. Pretende-se avaliar a contribuição efetiva das variáveis de clusterização nos agrupamentos formados.

\section{Referências}

ADLER, P. S.; CLARK, K. B. Behind the learning curve: a sketch of the learning process. Management Science, v. 37, n. 3, p. 267-281, 1991. http://dx.doi.org/10.1287/ mnsc.37.3.267

ANDERSON, J. R. Acquisition of cognitive skill. Psychological Review, v. 89, n. 4, p. 369-406, 1982. http://dx.doi.org/10.1037/0033-295X.89.4.369

ANZANELlO, M. J.; FOGLIATTO, F. S. Curvas de aprendizado: estado da arte e perspectivas de pesquisa. Gestão e Produção, v. 14, n. 1, p. 109-123, 2007a. http://dx.doi.org/10.1590/S0104-530X2007000100010

ANZANELLO, M. J.; FOGLIATTO, F. S. Learning curve modelling of work assignment in mass customized assembly lines. International Journal of Production Research, v. 45, n. 13, p. 2919-2938, 2007b. http:// dx.doi.org/10.1080/00207540600725010

ANZANELLO, M. J.; FOGLIATTO, F. S. Learning curve models and applications: literature review and research directions. International Journal of Industrial Ergonomics, v. 41, n. 5, p. 573-583, 2011a. http:// dx.doi.org/10.1016/j.ergon.2011.05.001

ANZANELLO, M. J.; FOGLIATTO, F. S. Programação de tarefas baseada em curvas de aprendizado para linhas de produção customizadas. Produção Online, v. 11, n. 3, 
p. 851-870, 2011b. http://dx.doi.org/10.14488/1676-1901. v11i3.955

BRIDA, J. G.; DISEGNA, M.; OSTI, L. Segmenting visitors of cultural events by motivation: a sequential non-linear clustering analysis of Italian Christmas Market visitors. Expert Systems with Applications, v. 39, n. 13, p. 11349-11356, 2012. http://dx.doi. org/10.1016/j.eswa.2012.03.041

DARDAN, S.; BUSCH, D.; SWARD, D. An application of the learning curve and the nonconstant-growth dividend model: IT investment valuation at Intel® Corporation. Decision Support Systems, v. 41, n. 4, p. 688-697, 2006. http://dx.doi.org/10.1016/j.dss.2004.10.004

DAR-EL, E. M.; RUBINOVITZ, J. Using learning theory in assembly lines for new products. International Journal of Production Economics, v. 25, n. 1-3, p. 103-109, 1991. http://dx.doi.org/10.1016/0925-5273(91)90136-H

ENGBLOM, J. et al. Multiple-method analysis of logistics costs. International Journal of Production Economics, v. 137, n. 1, p. 29-35, 2012. http://dx.doi.org/10.1016/j. ijpe.2012.01.007

EYRING, J. D.; JOHNSON, D. S.; FRANCIS, D. J. A cross-level units-of-analysis approach to individual differences in skill acquisition. Journal of Applied Psychology, v. 78, n. 5, p. 805-814, 1993. PMid:8253633. http://dx.doi.org/10.1037/0021-9010.78.5.805

FÁVERO, L. P. et al. Análise de dados: modelagem multivariada para tomada de decisões. Rio de Janeiro: Campus, 2009.

FIORETTI, G. The organizational learning curve. European Journal of Operational Research, v. 177, n. 3, p. 13751384, 2007. http://dx.doi.org/10.1016/j.ejor.2005.04.009

FOGLIATTO, F. S.; SILVEIRA, G. J. C.; BORESTEIN, D. The mass customization decade: an updated review of the literature. International Journal of Production Economics, v. 138, n. 1, p. 14-25, 2012. http://dx.doi. org/10.1016/j.ijpe.2012.03.002

GONG, S. et al. Multivariate analyses of element concentrations revealed the groupings of propolis from different regions in China. Food Chemistry, v. 134, n. 1, p. 583-588, 2012. http://dx.doi.org/10.1016/j. foodchem.2012.02.127

GUIMARÃES, L. B.; ANZANELLO, M. J.; RENNER, J. S. A learning curve-based method to implement multifunctional work teams in the Brazilian footwear sector. Applied Ergonomics, v. 43, n. 3, p. 541-547, 2012. PMid:21907970. http://dx.doi. org/10.1016/j.apergo.2011.08.008

HAIR JUNIOR, J. F. et al. Multivariate data analysis. Upper Saddle River: Prentice Hall, 1998.

JABER, M. Y.; BONNEY, M.; GUIFFRIDA, A. L. Coordinating a three-level supply chain with learningbased continuous improvement. International Journal of Production Economics, v. 127, n. 1, p. 27-38, 2010. http://dx.doi.org/10.1016/j.ijpe.2010.04.010

JABER, M. Y.; GLOCK, C. H. A learning curve for tasks with cognitive and motor elements. Computers \& Industrial Engineering, v. 64, n. 3, p. 866-871, 2013. http://dx.doi.org/10.1016/j.cie.2012.12.005

JOLLIFFE, I. T. Principal component analysis. 2nd ed. New York: Springer, 2002. (Springer Series in Statistics).
JUNG, U.; KOH, B.-H. Structural damage localization using wavelet-based silhouette statistics. Journal of Sound and Vibration, v. 321, n. 3-5, p. 590-604, 2009. http://dx.doi.org/10.1016/j.jsv.2008.10.016

LI, G.; RAJAGOPALAN, S. A learning curve model with knowledge depreciation. European Journal of Operational Research, v. 105, n. 1, p. 143-154, 1998. http://dx.doi.org/10.1016/S0377-2217(97)00033-7

MACCARTHY, B.; BRABAZON, P. G.; BRAMHAM, J. Fundamental modes of operation for mass customization. International Journal of Production Economics, v. 85, n. 3, p. 289-304, 2003. http://dx.doi.org/10.1016/ S0925-5273(03)00117-8

MAO, G.; HU, B.; SONG, H. Exploring talent flow in Wuhan automotive industry cluster at China. International Journal of Production Economics, v. 122, n. 1, p. 395-402, 2009. http://dx.doi.org/10.1016/j. ijpe.2009.06.008

MAZUR, J. E.; HASTIE, R. Learning as accumulation: a reexamination of the learning curve. Psychology Bulletin, v. 85 , n. 6, p. 1256-1274, 1978. PMid:734012. http:// dx.doi.org/10.1037/0033-2909.85.6.1256

NALDI, M. C. et al. Efficiency issues of evolutionary k-means. Applied Soft Computing, v. 11, n. 2, p. 1938 1952, 2011. http://dx.doi.org/10.1016/j.asoc.2010.06.010

NEMBHARD, D. A.; OSOTHSILP, N. Task complexity effects on between-individual learning/forgetting variability. International Journal of Industrial Ergonomics, v. 29, n. 5, p. 297-306, 2002. http:// dx.doi.org/10.1016/S0169-8141(01)00070-1

NEMBHARD, D. A.; UZUMERI, M. V. An individual-based description of learning within an organization. IEEE Transactions on Engineering Management, v. 47, n. 3, p. 370-378, 2000a. http://dx.doi.org/10.1109/17.865905

NEMBHARD, D. A.; UZUMERI, M. V. Experimental learning and forgetting for manual and cognitive tasks. International Journal of Industrial Ergonomics, v. 25, n. 4, p. 315-326, 2000b. http://dx.doi.org/10.1016/ S0169-8141(99)00021-9

PANANISWAMI, S.; BISHOP, R. C. Behavioral implications of the learning curve for production capacity analysis. International Journal of Production Economics, v. 24, n. 1-2, p. 157-163, 1991. http://dx.doi. org/10.1016/0925-5273(91)90162-M

PLAZA, M.; ROHLF, K. Learning and performance in ERP implementation projects: a learning-curve model for analyzing and managing consulting costs. International Journal of Production Economics, v. 115, n. 1, p. 72-85, 2008. http://dx.doi.org/10.1016/j. ijpe.2008.05.005

RENCHER, A. C. Methods of multivariate analysis. New York: John Wiley \& Sons, 1995.

RIED, S. A.; MIRKA, G. A. Learning curve analysis of a patient lift-assist device. Applied Ergonomics, v. 38, n. 6, p. 765-771, 2007. PMid:17194439. http://dx.doi. org/10.1016/j.apergo.2006.10.006

ROUSSEEUW, P. J. Silhouettes: a graphical aid to the interpretation and validation of cluster analysis. Journal of Computational and Applied Mathematics, v. 20, p. 53-65, 1987. http://dx.doi. org/10.1016/0377-0427(87)90125-7 
SAHA, I. et al. Improvement of new automatic differential fuzzy clustering using SVM classifier for microarray analysis. Expert Systems with Applications, v. 38, n. 12, p. 15122-15133, 2011. http://dx.doi.org/10.1016/j. eswa.2011.05.094

SEREL, D. A. et al. Investing in quality under autonomous and induced learning. IIE Transactions, v. 35 , p. 545-555, 2003. http://dx.doi. org/10.1080/07408170304415

SILVEIRA, G.; BORESTEIN, D.; FOGLIATTO, F. S. Mass customization: literature review and research directions. International Journal of Production Economics, v. 72, n. 1, p. 1-13, 2001. http://dx.doi.org/10.1016/ S0925-5273(00)00079-7

SMUNT, T. L.; WATTS, C. A. Improving operations planning with learning curves: overcoming the pitfalls of "messy" shop floor data. Journal of Operations Management, v. 21, n. 1, p. 93-107, 2003. http://dx.doi.org/10.1016/ S0272-6963(02)00088-8

STROIEKE, R. E.; FOGLIATTO, F. S.; ANZANELLO, M. J. Análise de conglomerados em curvas de aprendizado para formação de agrupamentos homogêneos de trabalhadores. Production, v. 23, n. 3, p. 537-547, 2013 a.

STROIEKE, R. E.; FOGLIATTO, F. S.; ANZANELLO, M. J. Estado da arte das aplicações de curvas de aprendizado. Gestão \& Produção, v. 20, n. 3, p. 681-694, 2013b. http://dx.doi.org/10.1590/S0104-530X2013000300013

TERWIESCH, C.; BOHN, R. E. Learning and process improvement during production ramp-up. International Journal of Production Economics, v. 70, n. 1, p. 1-19, 2001. http://dx.doi.org/10.1016/ S0925-5273(00)00045-1
TOWILL, D. R. Forecasting learning curves. International Journal of Forecasting, v. 6, n. 1, p. 25-38, 1990. http:// dx.doi.org/10.1016/0169-2070(90)90095-S

UZUMERI, M.; NEMBHARD, D. A population of learners: a new way to measure organizational learning. Journal of Operations Management, v. 16, n. 5, p. 515-528, 1998. http://dx.doi.org/10.1016/S0272-6963(97)00017-X

VITS, J.; GELDERS, L. Performance improvement theory. International Journal of Production Economics, v. 77, n. 3, p. 285-298, 2002. http://dx.doi.org/10.1016/ S0925-5273(00)00087-6

WONG, P. S. P.; CHEUNG, S. O.; HARDCASTLE, C. Embodying learning effect in performance prediction. Journal of Construction Engineering and Management, v. 133, n. 6, p. 474-482, 2007. http:// dx.doi.org/10.1061/(ASCE)0733-9364(2007)133:6(474)

WONG, P. S. P.; CHEUNG, S. O.; WU, R. T. H. Learning from project monitoring feedback: a case of optimizing behavior of contractors. International Journal of Project Management, v. 28, n. 5, p. 469-481, 2010. http://dx.doi.org/10.1016/j.ijproman.2009.07.003

WRIGHT, T. P. Factors affecting the cost of airplanes. Journal of the Aeronautical Sciences, v. 3, n. 4, p. 122-128, 1936. http://dx.doi.org/10.2514/8.155

YEH, S.; RUBIN, E. S. A review of uncertainties in technology experience curves. Energy Economics, v. 34, n. 3, p. 762-771, 2012. http://dx.doi.org/10.1016/j. eneco.2011.11.006

ZORGIOS, Y.; VLISMAS, O.; VENIERIS, G. A learning curve explanatory theory for team learning valuation. VINE, v. 39, n. 1, p. 20-39, 2009. http://dx.doi. org/10.1108/03055720910962425 


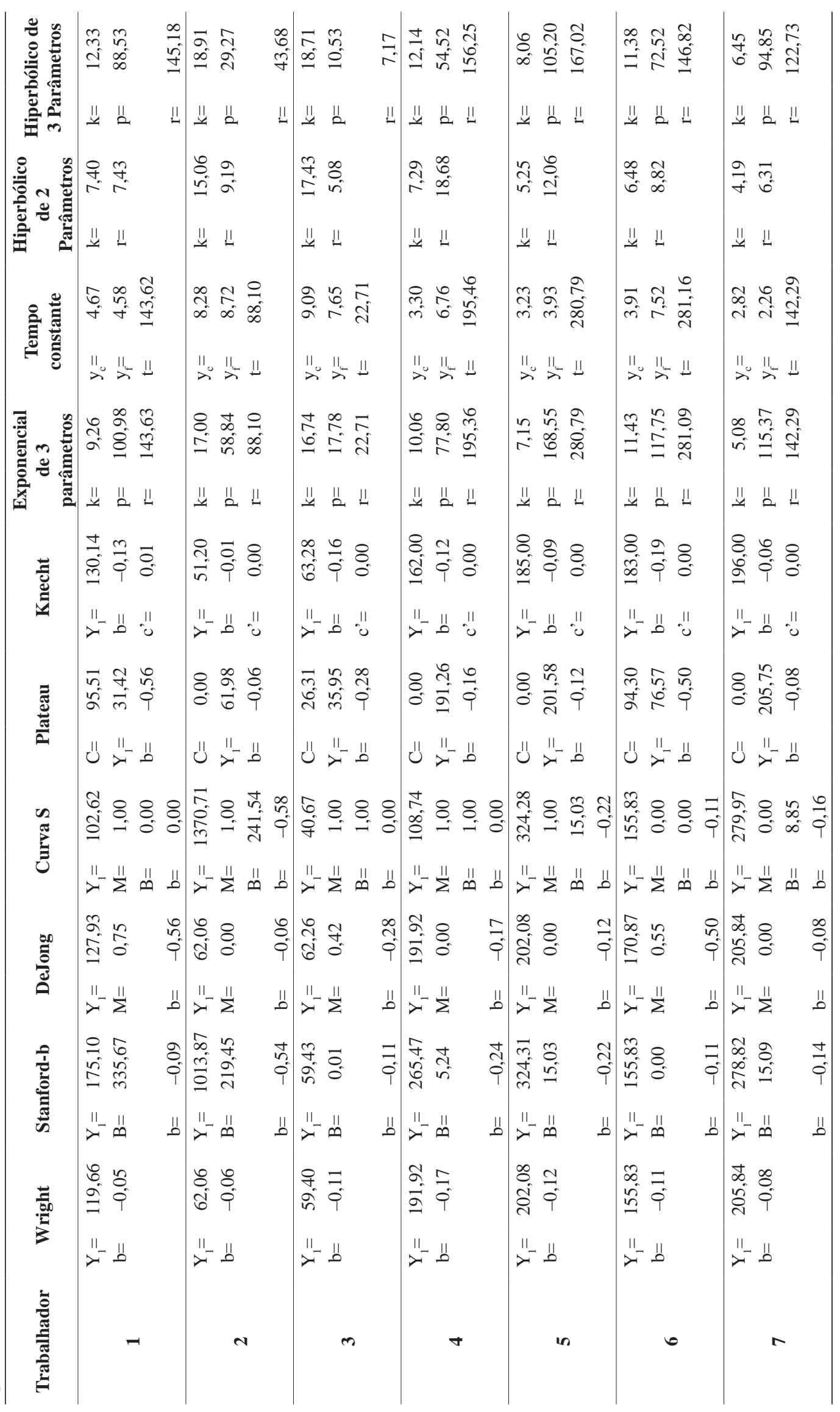




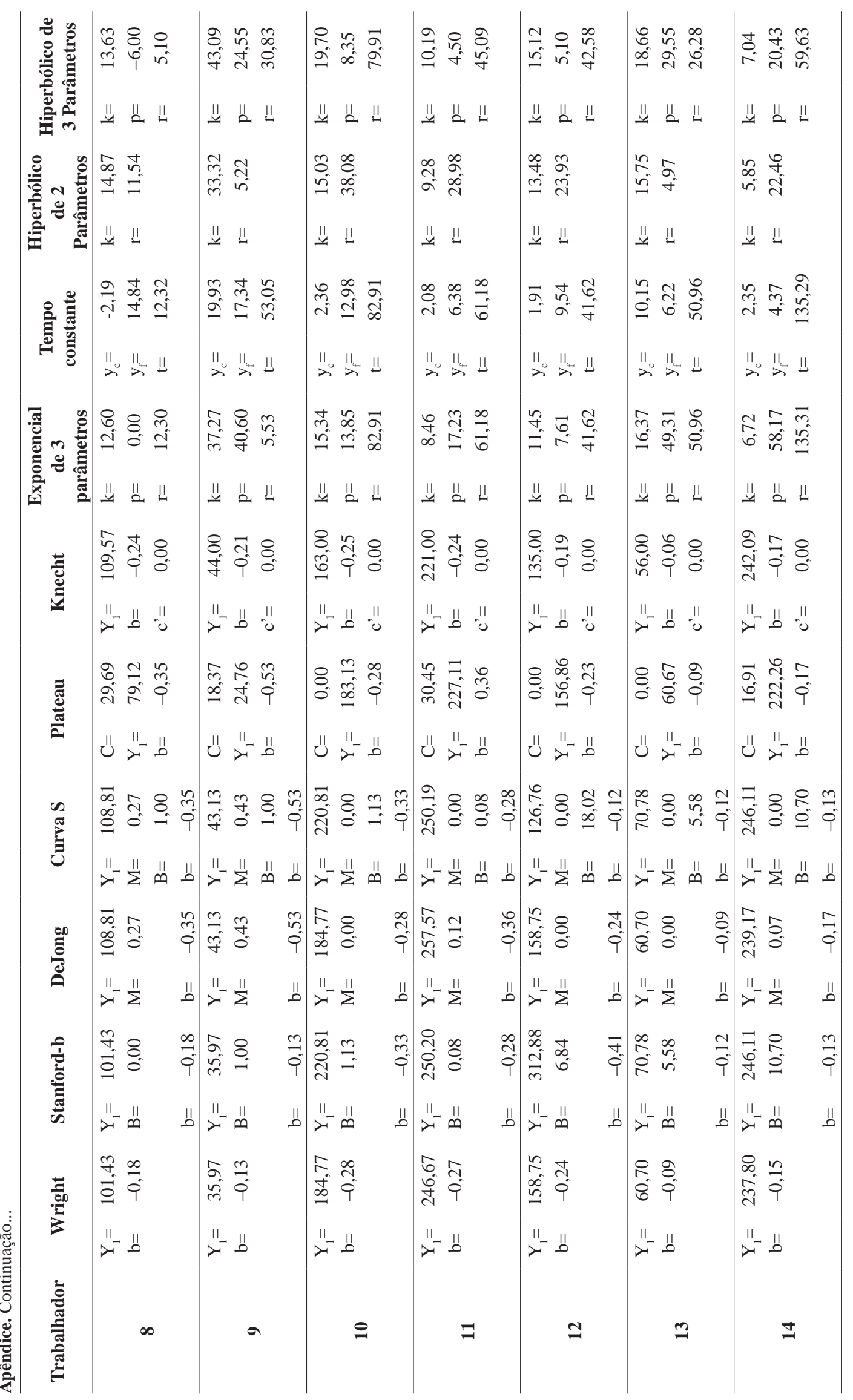




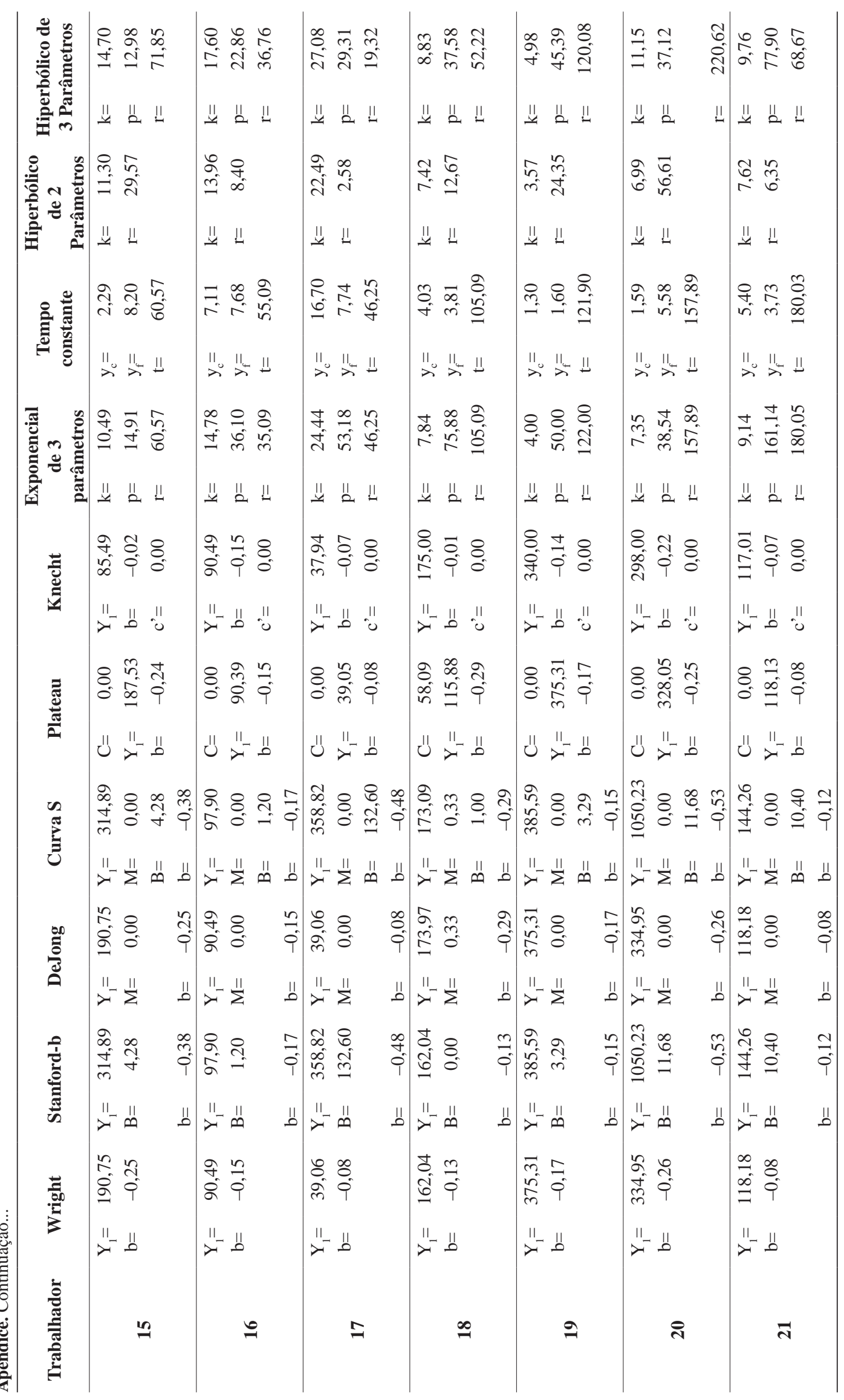

